\title{
COVID-19 2020: The Experience of a London Teaching Hospital's Nephrology Service
}

\author{
Elizabeth R. Wan ${ }^{a, b}$ Robert Unwin ${ }^{a, b}$ Stephen B. Walsh ${ }^{a, b}$ \\ aDepartment of Renal Medicine, Royal Free London NHS Foundation Trust, London, UK; b University College, \\ London, UK
}

2020 has been a year like no other, with the coronavirus pandemic leaving no one's life untouched. From February, we witnessed a dramatic increase in the number of cases across continents, which was soon followed by an explosion of COVID-19-related publications in almost all the leading medical journals. As we now move into 2021, it is worthwhile to look back at 2020 and the timeline in the evolution of our understanding of the virus' impact on day-to-day nephrology practice, mainly from the viewpoint of a hospital-based nephrologist still in training. What lessons have been learned and what should we take forward into our clinical practice to manage the current second and inevitable future waves of infection with this changing virus?

\section{What We Did Not Know at the Start of 2020?}

As a young nephrologist working in a large tertiary referral renal unit in London, I was aware of the events unfolding in Wuhan, China, at the end of 2019, but they seemed remote and of little relevance to my then clinical duties and practice. However, by March 2020 our hospital had rapidly built a "pod" in its car park for the expected
(C) 2021 The Author(s)

Published by S. Karger AG, Basel

This is an Open Access article licensed under the Creative Commons Attribution-NonCommercial-4.0 International License (CC BY-NC) (http://www.karger.com/Services/OpenAccessLicense), applicable to the online version of the article only. Usage and distribution for commercial purposes requires written permission. small number of cases of SARS-CoV-2 infection who would be tested and managed without risk to other hospital patients and staff. Within a few weeks, we had ambulances queuing up to deliver acutely ill patients to our Accident \& Emergency Department, and staff being called in urgently to fill emergency rotas. At the time I remember feeling desperate for information and guidance on how to manage these severely ill patients who presented with such alarming chest radiographs and frighteningly low oxygen saturation levels. In these early days, details about the infection and recommendations for its management were scarce and came largely by word-of-mouth. I recall the widespread use of a "WhatsApp" messaging group set up for doctors in London to exchange information and their experiences. An intensive care consultant with first-hand knowledge of managing lung involvement with COVID-19 was a valuable source of early guidance and recommended keeping these patients "dry" and not to give too much intravenous fluid. The reasoning behind this was that many COVID-19 infected patients appeared to develop a form of rapidly progressive acute respiratory distress syndrome (ARDS), and from prior experience of managing this condition, it had been shown that relying on intravenous fluids to provide 
haemodynamic support often made the situation in the lungs much worse. Indeed, I had first-hand experience of this in managing ARDS cases following the Grenfell Tower fire disaster in London in 2017 where many suffered smoke inhalation, and I was already very cautious about the use of intravenous fluids in such patients. We watched anxiously, while our patients struggled for breath in their isolated cubicles.

Within a few weeks, it was uninfected patients who were now placed in isolation to protect them from the increasing number of ward patients with COVID-19 infection. Our kidney transplant programme had been paused, COVID-19 began spreading rapidly throughout our dialysis units, and all the open bays on the inpatient wards were now filled with COVID-19 cases. Ambulatory care and outpatient visits were soon replaced by remote "telemedicine" consultations, which has proved more effective than expected and is likely to continue in some form even after the pandemic is over. We surveyed our inpatients with growing concern, while the television crews and reporters, and general public, observed us with increasing apprehension. Would we cope? We soon began seeing a worrying increase in cases of acute kidney injury (AKI), both on the general wards and in our intensive care unit (ICU). We wondered if we might have been running our patients too dry in restricting fluids to protect their lungs, and that perhaps we had underestimated their insensible losses from prolonged fever, or could it be due to the virus itself?

Back in 2005, it was already known that the spike protein of coronavirus causing SARS (severe ARDS), SARS$\mathrm{CoV}-1$, bound to the angiotensin-converting enzyme 2 (ACE2) to gain entry to infect cells [1], and this contributes to the lung injury seen [2]. This protein had been proposed as a vaccine target, and this is now bearing fruit [3]. ACE2 is expressed on the surface of type 2 alveolar epithelial cells, cardiac, and renal tissue. It acts as an important counter-regulator in the renin-angiotensin-aldosterone system by degrading ANGII to ANG1-7 and generating ANG1-9 peptide, which can bind to the cell-protective AT2 receptor and offset many of the adverse effects of AT1 stimulation [4]. When ANGII is degraded by ACE2, downstream effects include vasodilation, natriuresis, and lowered blood pressure. Knowing this, there has been a theoretical concern that patients treated with an ACE inhibitor (ACEi) or angiotensin receptor blocker (ARB) may be less or more susceptible to viral infection: ACEi or ARB treatment could potentially block viral entry, but treatment might also lead to increased tissue ACE2 expression that could be beneficial (see AT2 stimu- lation above) or might increase susceptibility to infection, as reviewed by South et al. [5]. Indeed, some have proposed AT2 receptor agonism as a treatment for COVID-19 to counter over-activation of RAS. A senior colleague in our department even began taking the ARB losartan at home as possible prophylaxis. Hypertension emerged as a risk factor for more severe COVID-19 infection but was it the hypertension or its treatment that predisposed to a worse infection? Additional concerns were the effects of a "cytokine storm" from overwhelming infection, as seen in multi-organ failure with immune dysregulation [6].

Very soon rapid prepublications began appearing that had not been peer-reviewed, but which began providing desperately needed initial data on the presentation and clinical features of infection and its early treatment. These publications were open access and made free by the journals that published them promptly online (including those that were peer-reviewed); this undoubtedly helped in sharing critical information quickly, provided experience that helped improve clinical management and was a basis for innovative clinical trials that have been conducted in "real-time" to determine best practice and effective therapies. However, the downside to this has been the widespread reporting of "off-label" therapies such as hydroxychloroquine as treatments, founded on very small observational studies or case reports, rather than proper randomized controlled trials [7]. This led to a coordinated push for so-called adaptive "platform" clinical trials in which repurposed and novel therapies could be tested in sequence and rapidly discarded or consolidated, for example, the RECOVERY trials based in Oxford, UK. This and similar, more formal, clinical trials soon invalidated hydroxychloroquine, showed that the antiviral remdesivir had some limited benefit and that the anti-inflammatory tocilizumab (anti-IL6 monoclonal antibody) did not fulfil its initial promise, as was also the case with convalescent plasma. Only dexamethasone (so far) has been shown to reduce mortality in those patients requiring oxygen [8]. More trials of proposed treatments are still ongoing.

Cheng et al. [9] published one of the first retrospective case series of 1,300 COVID-19 patients in China and reviewed, amongst other things, the incidence of AKI. Patients with diabetes and hypertension seemed to be more at risk of developing AKI, and those with AKI did much worse. In fact, patients with COVID-19 and AKI had a higher mortality than non-COVID-19 critically ill patients with AKI. Haematuria and proteinuria were common findings. Although many of these patients had been 
given diuretics (to manage their pulmonary and cardiac complications), perhaps there was something more than dehydration and not only a prerenal cause? We started to plan for large numbers of patients who might need longterm renal replacement therapy after discharge from hospital, but unfortunately few survived their admission to the ICU. The first post-mortem series of COVID-19 cases shed some light on what was happening. Twenty-six patients in one series underwent an autopsy, of which 9 had AKI [10]. In these 9 cases, light microscopy showed proximal tubular injury, and 3 of these had cast in keeping with rhabdomyolysis. Electron microscopy showed viral particles within tubular cells although this has since been questioned [11].

A review of cases in New Orleans identified 5 phenotypes of AKI in COVID-19, the predominant one being acute tubular injury (66\%) [12]. At our hospital, we had also noted that patients on continuous veno-venous haemofiltration $(\mathrm{CVVH})$ circuits had significant clotting problems, and many were found to have pulmonary emboli, which raised concerns about an associated coagulopathy and microangiopathy in the kidney. The haematuria and proteinuria suggested glomerular injury, and we know now that haematuria and proteinuria predict more likely progression to critical illness [13]. Rhabdomyolysis, which we did see, was less common and was seen in only $7 \%$ of AKI cases in the New Orleans cohort.

The relatively low incidence of AKI reported in Chinese cohorts determined service planning and provision in London. The new London Nightingale Hospital, which was set up as a military style hospital in record time, was an impressive example of emergency planning and construction, but it was set up without any CVVH provision or a dialysis water supply. Subsequent publications of cohorts in the USA matched more closely the London experience of AKI incidence in COVID-19 [14, 15]. Information sharing amongst nephrology units within and outside of London proved very helpful, for example, a single centre experience of placing patients in a prone position for acute peritoneal dialysis, or linking up several patients to share a single CVVH machine.

As the pandemic continued to ravage our existing cohort of renal patients, collaborative efforts such as the ERACODA (European Renal Association COVID-19 Database) confirmed that the predominant risk factors for poor outcomes were common between dialysis patients, transplant recipients, and the general population, especially advanced age and frailty [16]. Subsequent retrospective analysis of our local in-centre haemodialysis cohort has identified additional features associated with greater risk for hospital admission and mortality, including a high neutrophil-to-lymphocyte ratio and C-reactive protein on laboratory testing [17]. Early local challenges with this specific patient group included provision of safe, isolated hospital transport. Dialysis transport is frequently quoted as making up 50\% of non-emergency patient transport paid for by the National Health Service. Our local transport provider declined to take confirmed COVID-19 patients to dialysis, so this need had to be met by charitable organisations such as St John's Ambulance and Hatzola. Their selfless efforts were much appreciated; fortunately, transportation problems had been largely resolved by the second wave. We have ongoing difficulties with availability of isolation bays in the dialysis units, and I wonder if the pandemic will affect design of future haemodialysis units, and perhaps even the programmes themselves (with a shift towards home therapies).

Case reports started to emerge of other kidney-related disease phenomena. From New York came reports of a collapsing glomerulopathy (GN) in patients of African ancestry [18], subsequently linked to carriage of the APOL1 risk alleles known to predispose to HIVAN and FSGS, as well as CKD, and probably linked to viral-triggered interferons. In fact, interferon-beta was being proposed and used as antiviral therapy in some cases, although mainly in China, and also by inhalation for ARDS. Other renal biopsy series began to show more diverse glomerular phenotypes [19], suggesting that direct viral invasion may be less important than immune-mediated damage; in our own unit, we saw relapse in a patient with pre-existing membranous nephropathy. One of our transplant patients developed secondary haemophagocytic lymphohistiocytosis, a rare, severe, and often fatal systemic inflammatory condition seen more usually in patients with pre-existing chronic inflammatory diseases or a primary immunodeficiency; the patient responded to treatment with the IL-1 receptor antagonist Anakinra. Interestingly, evidence for a direct tubular insult persisted; we identified a patient with a transient renal Fanconi syndrome following SARS-CoV-2 infection with low molecular weight proteinuria [20].

\section{What Do We Know at the End of 2020?}

As the second wave of infections brings another long line of ambulances to our hospital door, the ICU is once again overflowing, and the transplantation programme is once more on hold; it is easy to have a sense of deja $v u$ and to feel despair at the prospect of a repeat of what hap- 
pened in March 2020. Has nothing changed? However, it is important to remember that we do have almost 12 months of experience to draw on.

Animal work suggests that renin-angiotensin-aldosterone system blockade is not associated with upregulation of ACE2 [21], and analysis of large cohorts now suggests that the association between hypertension and severe disease is not as strong as initially feared [22]. Advice is to continue prescribing ACEi/ARB therapy to inpatients already taking them, and we await the results of the BRACECORONA trial comparing maintenance with withdrawal of ACEi/ARB therapy in patients with COVID-19. Common sense suggests maintaining euvolaemia with intravenous fluids is the best approach to patient management and to avoid fluid overload by taking account of insensible losses. Thanks to the RECOVERY trial, we now know that dexamethasone is beneficial for hypoxic patients although we do not know yet whether it can also reduce development of AKI and the need for renal replacement therapy. Remdesivir may be helpful in some patients, but its use in renal patients may prove challenging [23]. Again, we also know what treatments are unlikely to helpful, for example, lopinavir-ritonavir, and in some instances might even be harmful, for example, hydroxychloroquine.

Concerning renal transplantation, there has been intense discussion as to whether there is an increased risk of infection. Initially, it was thought that immunosuppressive drugs like cyclosporin and tacrolimus might offer some protection because of their antiviral properties [24] but fears that immunosuppression may increase susceptibility to infection lead to the approach that immunosuppressive drugs should be minimized as safely as possible. Reports have now suggested that infection risk in renal transplant patients is no different from the general population when taking into account age and other predisposing comorbidities like diabetes and obesity [25]. We are currently withholding antiproliferative agents when transplant recipients are diagnosed with (or suspected of having) COVID-19, in keeping with guidance from the British Transplantation Society and Renal Association [26]. Outcomes have been largely favourable for this patient group, who are generally younger and less frail than haemodialysis patients. Both transplanted and dialysis patients are considered high priority for vaccination although based more on their associated comorbidities; while vaccine efficacy may be reduced, the risk of triggering rejection is thought to be low for any of the currently licensed vaccines (none is live-attenuated).
A worrying development is the emergence of longterm effects from COVID-19 infection, so-called long COVID syndrome, which may, in fact, be as many as 4 different syndromes, including post-intensive care and post-viral fatigue syndromes [27]. CKD is another sequela that is becoming recognized, and one to watch out for, especially in those who experienced an episode of AKI.

During this time, we are also reminded of earlier pandemics, especially the 1918 flu pandemic known as "Spanish flu" (a historical misnomer: it was first described in USA military personnel and may have been brought over to Europe when the USA joined the First World War in 1917). Perhaps not surprisingly, there are some interesting parallels with debates over the origins of the infection and then response to the pandemic, especially the early efforts made to limit its spread, including isolation, travel restrictions, and the wearing of masks. At the time a bacterial, rather than a viral, cause was suspected (haemophilus influenzae in particular, hence its name); those most affected were young and died of what was almost certainly a severe form ARDS during the first wave of infections in the spring of 1918. But haemophilus was not causative, although secondary bacterial pneumonia was the more likely cause of death during the second, more severe wave of infections in the autumn of the same year [28]. Antibiotics were not available; passive immunisation was tried with antisera raised against haemophilus, as well as vaccines against pneumococcus, but these had only limited benefit. However, there was more success with giving plasma taken from convalescent patients, another parallel with approaches used today. Long-term consequences of this infection also emerged a decade or so later, the most striking being a possible link to a form of encephalitis, "encephalitis lethargica," highlighted in the 1997 book Awakenings by Oliver Sacks. Now, with our new medical knowledge and experience, we have a clearer idea of what clinical services need to be adequately resourced, as well as the prospect of new vaccines being rolled out: an astonishing scientific achievement in such a short time.

Can we keep ahead of this virus and plan better for future pandemics, which seem likely to occur? Only time will tell, but perhaps we can begin 2021 with a little more optimism, show even more concern for our environment, and salute the extraordinary mobilization and cooperation of the worldwide scientific, pharmaceutical, and healthcare communities in tackling COVID-19. 


\section{Acknowledgements}

The authors are grateful for the support and commitment of nephrologists both in London and worldwide over the past year.

\section{Conflict of Interest Statementt}

E.R.W. and S.B.W. have no COI to report. R.J.U. is currently employed by AstraZeneca Biopharmaceutical R\&D, Cambridge UK and Gothenburg Sweden.

\section{Funding Sources}

This work was not funded.

\section{Author Contributions}

E.R.W. wrote the piece; R.J.U. contributed. S.B.W. reviewed and assisted with revisions.

\section{References}

1 Chu KH, Tsang WK, Tang CS, Lam MF, Lai $\mathrm{FM}$, To KF, et al. Acute renal impairment in coronavirus-associated severe acute respiratory syndrome. Kidney Int. 2005;67(2):698705.

2 Kuba K, Imai Y, Rao S, Gao H, Guo F, Guan $\mathrm{B}$, et al. A crucial role of angiotensin converting enzyme 2 (ACE2) in SARS coronavirusinduced lung injury. Nat Med. 2005;11(8): 875-9.

3 Du L, He Y, Zhou Y, Liu S, Zheng BJ, Jiang S. The spike protein of SARS-CoV-a target for vaccine and therapeutic development. Nat Rev Microbiol. 2009;7(3):226-36.

4 Ocaranza MP, Jalil JE. Protective role of the ACE2/Ang-(19) axis in cardiovascular remodeling. Int J Hypertens. 2012;2012.

5 South AM, Tomlinson L, Edmonston D, Hiremath S, Sparks MA. Controversies of renin-angiotensin system inhibition during the COVID-19 pandemic. Nat Rev Nephrol. 2020;16(6):305-7.

6 Qin C, Zhou L, Hu Z, Zhang S, Yang S, Tao Y, et al. Dysregulation of immune response in patients with coronavirus 2019 (COVID-19) in Wuhan, China. Clin Infect Dis. 2020; 71(15):762-8.

7 Saag MS. Effect of Hydroxychloroquine in Hospitalized Patients with Covid-19. JAMA. 2020;383:2030-40.

8 Group TRC. Dexamethasone in hospitalized patients with Covid-19 - preliminary report. N Engl J Med. 2020:1-11.

9 Cheng Y, Luo R, Wang K, Zhang M, Wang Z, Dong L, et al. Kidney disease is associated with in-hospital death of patients with COVID-19. Kidney Int. 2020;97(5):829-38.

10 Su H, Yang M, Wan C, Yi LX, Tang F, Zhu HY, et al. Renal histopathological analysis of 26 postmortem findings of patients with $\mathrm{CO}-$ VID-19 in China. Kidney Int. Published Online First. 2020
11 Calomeni E, Satoskar A, Ayoub I, Brodsky S, Rovin BH, Nadasdy T. Multivesicular bodies mimicking SARS-CoV-2 in patients without COVID-19. Kidney Int. 2020;98(1):233-4.

12 Mohamed MMB, Lukitsch I, Torres-Ortiz AE, Walker JB, Varghese V, Hernandez-Arroyo $\mathrm{CF}$, et al. Acute kidney injury associated with coronavirus disease 2019 in urban New Orleans. Kidney360. 2020;1(7):614-22.

13 Zheng X, Yang H, Li X, Li H, Xu L, Yu Q, et al. Prevalence of kidney injury and associations with critical illness and death in patients with COVID-19. Clin J Am Soc Nephrol. 2020;15(11):1549-56.

14 Chan L, Chaudhary K, Saha A, Chauhan K, Vaid A, Zhao S, et al. AKI in Hospitalized Patients with COVID-19. J Am Soc Nephrol. 2021 Jan;32(1):151-160.

15 Hirsch JS, Ng JH, Ross DW, Sharma P, Shah $\mathrm{HH}$, Barnett RL, et al. Acute kidney injury in patients hospitalized with COVID-19. Kidney Int. 2020;98(1):209-18.

16 Hilbrands LB, Duivenvoorden R, Vart P, Franssen CFM, Hemmelder MH, Jager KJ, et al. COVID-19-related mortality in kidney transplant and dialysis patients: Results of the ERACODA collaboration. Nephrol Dial Transplant. 2020;35(11):1973-83.

17 Hendra H, Vajgel G, Antonelou M, Neradova A, Manson B. Identifying prognostic risk factors for poor outcome following COVID - 19 disease among in - centre haemodialysis patients: role of inflammation and frailty. J Nephrol. Published Online First. 2021.

$18 \mathrm{Wu} \mathrm{H}$, Larsen CP, Hernandez-Arroyo CF, Mohamed MMB, Caza T, Sharshir M, et al. AKI and collapsing glomerulopathy associated with covid-19 and apol1 high-risk genotype. J Am Soc Nephrol. 2020;31(8):1688-95.

19 Kudose S, Batal I, Santoriello D, Xu K, Barasch J, Peleg Y, et al. Kidney biopsy findings in patients with COVID-19. J Am Soc Nephrol. 2020;31(9):1959-68.
20 Wan ER, Woolfson RG, Greenwood R, Walsh SB. Transient renal tubular syndromes associated with acute COVID-19 disease. Kidney Int Rep. 2020;5(9):1610-1.

21 Wysocki J, Lores E, Ye M, Soler MJ, Batlle D. Kidney and lung ACE2 expression after an ACE inhibitor or an ang II receptor blocker: implications for COVID-19. J Am Soc Nephrol. 2020;31(9):1941-3.

22 Petrilli CM, Jones SA, Yang J, Rajagopalan H, O’Donnell L, Chernyak Y, et al. Cerfolio, Fritz Francois LIH. Factors associated with hospitalization and critical illness among 4,103 patients with Covid-19 disease in New York City. medRxiv. 2020.

23 Adamsick ML, Gandhi RG, Bidell MR, Elshaboury RH, Bhattacharyya RP, Kim AY, et al. Remdesivir in patients with acute or chronic kidney disease and COVID-19. J Am Soc Nephrol. 2020;31(7):1384-6.

24 Willicombe M, Thomas D, Mcadoo S. COVID-19 and calcineurin inhibitors : should they get left out in the storm? JASN. 2020:1145-6.

25 Devresse A, Belkhir L, Vo B, Ghaye B, Scohy A, Kabamba B, et al. COVID-19 infection in kidney transplant recipients: a single-center case series of 22 cases from belgium. Kidney Med. 2020 Jul-Aug;2(4):459-66.

26 BTS, The Renal Association. Guidance on the management of transplant recipients diagnosed with or suspected of having COVID19. 2020. https://www.who.int/publications-detail/clinical-management-of-severe-acuterespiratory.

27 Maxwell E. Living with Covid-19. NIHR 2020;31:197-200.

28 Morens DM, Taubenberger JK, Fauci AS. Predominant role of bacterial pneumonia as a cause of death in pandemic influenza: Implications for pandemic influenza preparedness. J Infect Dis. 2008;198(7):962-70. 